\title{
Bridging Biomedical and Person-centered Care Approaches via Namaste Care with Family Participation: An Asian Experience in Advanced Dementia Treatment
}

Noorhazlina Ali ${ }^{1,2,}{ }^{*}$, Cai Ning Tan ${ }^{2}$, Jasmine Kang ${ }^{4}$, Aik Phon Chew ${ }^{1,2}$, Caroline Caroline ${ }^{2,3}$, Mary Lee $^{5}$, Wee Shiong Lim $^{1,2}$

1. Department of Geriatric Medicine, Tan Tock Seng Hospital, Singapore; E-Mails: noorhazlina@ttsh.com.sg; Aik Phon Chew@ttsh.com.sg; Wee Shiong Lim@ttsh.com.sg

2. Institute of Geriatrics and Active Ageing, Singapore; E-Mail: Cai Ning TAN@ttsh.com.sg

3. Department of Continuing and Community Care, Tan Tock Seng Hospital, Singapore; E-Mail: caroline 1@ttsh.com.sg

4. Nursing Service, Tan Tock Seng Hospital, Singapore; E-Mail: Jasmine Kang@ttsh.com.sg

5. Health Outcomes and Medical Education Research, National Healthcare Group, Singapore; EMail: Mary Cl LEE@nhg.com.sg

* Correspondence: Noorhazlina Ali; E-Mail: noorhazlina@ttsh.com.sg

Academic Editor: Ladislav Volicer

Special Issue: Advanced Dementia: Enjoy It or Avoid It?

OBM Geriatrics

2020, volume 4 , issue 4

doi:10.21926/obm.geriatr.2004140
Received: October 27, 2020

Accepted: November 23, 2020

Published: December 04, 2020

\begin{abstract}
A locally-adapted, multisensory, psychosocial intervention called Namaste Care program was developed to improve the daily life of persons with advanced dementia (PWAD) through arranging meaningful activities and physical interactions by healthcare staff (Namaste Carers). Congruent with Asian values, the families of PWAD were invited to participate in Namaste Care sessions. The study aimed to explore the influence of Namaste Care on cognitive status and quality of life (QOL) of PwAD, and perceptions and attitudes of caregivers and Namaste Carers toward dementia care were determined. A total of 10
\end{abstract}

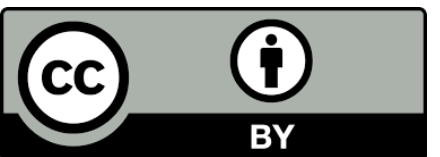

(C) 2020 by the author. This is an open access article distributed under the conditions of the Creative Commons by Attribution License, which permits unrestricted use, distribution, and reproduction in any medium or format, provided the original work is correctly cited. 
individuals, including patient-caregiver dyads $(n=4)$ and Namaste Carers $(n=6)$ participated in a program in a tertiary hospital in Singapore. Quality of Life in Late-Stage Dementia (QUALID) scale and Severe Impairment Rating Scale (SIRS) were employed to evaluate QOL and cognition, respectively, in the pre- and post-survey program in patient-caregiver dyads. Namaste Carers' knowledge and attitudes toward PwAD were assessed using the Questionnaire on Palliative Care for Advanced Dementia (qPAD). Caregivers and Namaste carers were interviewed post-program separately. A concurrent explanatory mixed-method analysis was done. The mean age of PwAD was 84 years, with $75 \%(n=3)$ patients on enteral tube feeding. Namaste carers were nurses with an average experience of 4.1 years in dementia care. On comparison of pre- and post-program scores of PWAD, QUALID scores showed a decrease (indicating an improvement in the QOL), while the SIRS scores were increased (indicating an improved cognitive response). Namaste Carers scored high on qPAD, reflecting the good understanding and a positive attitude toward PWAD. Thematic analysis of 10 interview transcripts from caregivers and Namaste Carers revealed three themes: the polarizing paradigm of care in advanced dementia; pre-eminence of the Asian family values, and theme of "small actions make a big difference". Namaste Care served as a bridge between the person-centered care approach and the biomedical model of dementia care. Namaste Care with family participation may be the cornerstone of PWAD to receive culturally-appropriate personalized care and serve as a premise for the operationalization of person-centered care in Asian societies, and undeniably across the world.

\section{Keywords}

Advanced dementia; Namaste Care; person-centered care; quality of life; quality care; persons with dementia; family caregivers

\section{Introduction}

The provision of dementia care spans a continuum along the disease trajectory, with care at the advanced dementia stage focusing on the preservation of dignity, when preservation of independence becomes a priority. Owing to severe decline in cognitive faculty and functional ability, persons with advanced dementia (PwAD) enjoy limited personal autonomy and engagement in care delivery. The traditional paradigm of the biomedical model of dementia care assumes linear causality between neuropathology and dementia [1], with a focus on medical diagnosis and treatment. This model led to the medicalization of dementia in which the illness is not viewed within the context of the social system [2]. In contrast, underpinned by the personcentered care framework, the biopsychosocial approach to dementia care emphasizes understanding the actual persons behind the illness, addressing their needs holistically, and improving their QOL $[3,4]$. The gap between biomedical-centric and person-centric care approaches is especially noteworthy in advanced dementia, leading to renewed calls to bridge it [5, $6]$.

Indeed, person-centered care is germane to the PWAD and can be viewed as a proxy indicator of quality care. It is also widely viewed as the central tenet of good quality dementia care and 
embodies empowering, respectful, holistic, and individualized care [7]. Such personalized quality care is provided to the PWAD through Namaste Care-a multisensory and psychosocial intervention program for the PWAD-reported to create opportunities for delivery of person-centered care $[8,9]$ and is structured to fulfill the psychological needs of the PwAD [10].

The multitude of benefits of Namaste care reported in the literature range from a reduction of antipsychotics use $[11,12]$ and severity of behavioral and psychological symptoms of dementia [13, 14] to improving quality of care and life for $\operatorname{PWAD}[15,16]$. Being a multi-component intervention, Namaste Care gives freedom to carers in delivering tailored interventions to PwAD by implementing different multiple components (touch therapy, massage, aromatherapy, music therapy, doll therapy, etc.) in the same session depending on the preferences and needs of PwAD. These components of the program are reported to have proven efficacy in PWAD [17]. Through Namaste Care, person-centered care approaches are deployed via adherence to the principles of loving touch and a calm environment [18]. Being a multidimensional care program, it is aligned with several key dimensions of person-centered care, such as biopsychosocial perspective, patient-as-person, and therapeutic alliance [19]. It encourages shifting from the biomedical perspective to the biopsychosocial one while establishing a relationship-based culture of care.

While person-centered care ought to be delivered in all care settings, translation and implementation of such practice to the ground is highly variable and is often dependent on the care delivery setting. Reports of conduct and attendant benefits of Namaste Care are mainly focused on long term care settings or nursing homes [20-26]. There is a dearth of studies on Namaste Care for PWAD in the acute setting or step-down care facilities such as subacute wards and community hospitals. Notwithstanding, there is immense potential in introducing Namaste Care in the hospital environment [27] where person-centered care needs of PWAD can be met despite higher acuity of care rendered.

The development of Namaste Care in Asian countries is slowly gaining attraction and currently, there is a scarcity of scientific publications showing the effect of Namaste Care on Asian PwAD and their caregivers. Recent studies [9, 11-15, 18, 20-21, 24-27] have predominantly been centered on European and North American countries. Namaste Care may be viewed as a vehicle through which person-centered and culturally sensitive care is delivered. The latter requires negotiation of traditional Asian values, with a strong emphasis on the family's involvement in healthcare decisions while respecting the individual's choice [28]. The person-centered care values embodied in Namaste Care are congruent with how some Asian families perceive themselves to be an integral part of the care team [29]. One's culture, beliefs, and values influence the way dementia care or PWAD is perceived [30] and also the way to develop culturally congruent interventions or strategies to deliver person-centered care. Proper understanding of the nuances behind the diverse cultural beliefs and perceptions provides ample opportunities to tailor interventions or strategies for dementia care.

The primary objective of this mixed-methods study was to explore the influence of locallyadapted Namaste Care program on the cognitive status and quality of life of PWAD, and the perceptions and attitudes of Asian caregivers and healthcare staff toward dementia care in PWAD. 


\section{Methods}

\subsection{Setting, Participants, and Recruitment}

This study was conducted in a step-down unit in a tertiary hospital in Singapore between May 2015 and April 2017. The step-down unit was specialized in sub-acute care and had patients undergoing continuation of medical treatment beyond the acute phase of illness, short course rehabilitation, or in the coordination of discharge planning.

The Namaste Care program for PwAD in the step-down unit was modified to suit the local healthcare setting and the social and cultural norms. It was made available to all PWAD who were admitted or transferred to the step-down unit. Namaste Care was provided by healthcare staff (Namaste Carers) who had volunteered to participate in the program. These volunteers underwent two hours of a didactic and interactive training session on complications in advanced dementia and on the underlying principle, purpose, and conduct of Namaste Care. They were also taught various methods of delivering meaningful activities to PwAD through the program. The program was scheduled to coincide with the two-hour nursing change-of-shift period (morning shift: 0700 h-1530 h; afternoon shift: 1300 h-2130 h) when manpower was adequately available. A roster was developed to assign Namaste Carers to duty during their shift. Whenever manpower constraints were experienced, Namaste Carers adjusted the timing of the program and volunteered to deliver the Namaste Care session beyond their shift. The subsequent involvement of non-medical volunteers, trained as Namaste Carers, ensured the sustainability of the program as protected/extended time was not consistently conferred to healthcare staff conducting Namaste Care. These Namaste Carers engaged daily with the PWAD for two hours in the afternoon, seven days a week, during the course of their stay in the unit. On average, one to two PWAD participated in Namaste Care sessions conducted by one Namaste Carer, though a maximum of four PWAD were allowed to participate in the program per session. The sensory-based program incorporated two principles of Namaste Care, namely creating a peaceful environment and providing activities and healthy interactions with an unhurried and loving approach. The room designated for Namaste Care sessions was transformed into a familiar home-like environment. It was decorated with wall and ceiling decals, curtains, paintings, and plants. The activities were locally adapted through the infusion of Asian sacred elements, such as the use of traditional Asian spices and scents for aromatherapy. The locally-adapted program invited and encouraged healthy participation by family caregivers alongside in collaboration with Namaste Carers. This is a novel approach vies-a-vies the original version of the Namaste Care program [8], where family involvement remains focused on regular family meetings to discuss meaningful activities and quality of life care.

The inclusion criteria for patient-caregiver dyads were diagnosis with advanced dementia (defined as at least Functional Assessment Staging Tool (FAST) stage 7 [31]) and the presence of English-speaking legally acceptable representatives (family caregivers). The delirious, disoriented or medically unstable patients were excluded. Family caregivers were first approached for assent to the study on behalf of PWAD, who were found incapable of providing consent. Written consent was then obtained from family caregivers who were the legally acceptable representatives of PWAD, and from healthcare staff/the Namaste Carers. 
Four patient-caregiver dyads and six Namaste Carers participated in the study. The study was approved by the Domain Specific Review Board (DSRB), National Healthcare Group, Singapore (NHG DSRB Ref: 2015/00175).

\subsection{Procedure and Data Collection}

The data on baseline characteristics (age, gender, ethnicity, and extent of comorbidities) of the participants were collected using the Charlson Comorbidity Index [32]. Demographics of caregivers (co-residency status, patient-caregiver relationship) and Namaste Carers (education level, employment status, working experience with persons with advanced dementia) were also gathered via carer-administered questionnaires.

The cognitive status of the PWAD was assessed using the Severe Impairment Rating Scale (SIRS) at baseline and just before discharge from the step-down unit. Caregivers were surveyed before and after the program on the QOL of PWAD using the QUALID scale. Similarly, the Namaste Carers were assessed on their knowledge and attitudes toward PWAD via a Questionnaire on Palliative Care for Advanced Dementia (qPAD).

Severe Impairment Rating Scale (SIRS): SIRS is a locally validated cognitive assessment tool to measure the residual cognitive ability of PWAD and comprises 14 items [33, 34]. Scores range from 0 to 28 , with higher scores indicating better cognitive functioning.

Quality of Life in Late-Stage Dementia (QUALID): QUALID is a dementia-specific scale and is administered in an interview format to the caregivers [35]. The scoring is done by summing the responses, with possible scores ranging from 11 to 55. The lowest score of 11 represents the highest QOL.

Questionnaire on Palliative Care for Advanced Dementia ( $9 P A D)$ : qPAD assesses Namaste Carers' knowledge, belief, perceptions, and attitudes about palliative and End-of-Life care for the PwAD [36, 37]. It consists of two parts: a 23-item Knowledge Test and a 12-item Attitudes Scale. Knowledge Test consists of three subscales (measuring anticipating needs, prevention of negative outcomes, intuition, and insight) and is answered through Agree, Disagree, and Do not Know responses. The total score ranges from 0 to 23, with higher scores indicating greater knowledge on palliative care for advanced dementia. The Attitudes Scale consists of three subscales (i.e., job satisfaction; perceptions and belief; work setting support of families) and is a 5-point Likert scale where responses are registered from 1 (strongly disagree) to 5 (strongly agree). The total score ranges from 12 to 60 , with a higher score signifying greater positive attitudes toward palliative care for advanced dementia.

A one-on-one focused semi-structured interview was conducted in the English language, at least one week-post program on caregivers and Namaste Carers to elicit their feelings, attitudes, and perceptions of PWAD and to evaluate the impact of the Namaste Care program on them. These audiotaped interviews were then transcribed verbatim and anonymized before analysis.

\subsection{Analysis}

A concurrent explanatory mixed-method analysis was conducted. For quantitative analysis, we performed descriptive statistics to derive means and standard deviations for the continuous variables and percentages for categorical variables for baseline demographics and clinical characteristics of patient-caregiver dyads and Namaste Carers, as well as pre- and post- outcome 
measures. As this was an exploratory study in a small sample, inferential statistics were not performed.

The qualitative data from the transcribed audiotaped interviews were analyzed using thematic analysis, a form of a qualitative analytic method [38]. Immersion of the data was conducted by two members of the study team (NA and $M L$ ), and initial codes were generated prior to formal coding, an inclusive and comprehensive process. Through an inductive approach, these codes were analyzed independently and then raised/matched to themes and sub-themes. The themes were reviewed and refined jointly iteratively until a consensus was reached to ensure coherence with the dataset.

Both quantitative and qualitative datasets were merged to enable an integrated view of the influence of Namaste Care on the patient-caregiver dyads and Namaste Carers. This merger of datasets enabled complementarity through simultaneous interrogation of quantitative and qualitative data with equal weightage to result in a deeper analysis and interpretation of the phenomenon.

\section{Results}

\subsection{Quantitative Results}

Four patient-caregiver dyads and six Namaste Carers participated and completed the study (see Table 1). The mean age of PwAD was 84 years, with the majority of them being females $(n=3)$ and of Chinese ethnicity. Baseline reduced cognitive abilities were reflected by the low mean SIRS score of 2.0 (SD = 1.9). The PWAD had multiple comorbidities, and $75 \%$ of them were on enteral tube feeding. All the caregivers were adult children, highly literate, and $50 \%(n=2)$ of them had participated in the locally-adapted Namaste Care program alongside Namaste Carers. The Namaste Carers were nurses of varying seniority with an average experience of 4.1 years in dementia care settings.

Table 1 Baseline characteristics of the patient-caregiver dyads and Namaste Carers.

\begin{tabular}{lll}
\hline \multicolumn{2}{c}{ Characteristics } \\
\hline & Age (years) & $84.0 \pm 4.7$ \\
& Female (\%) & 75.0 \\
& Race (\%) & \\
& Chinese & 75.0 \\
& Indian & 25.0 \\
& Charlson Comorbidity Index & $6.0 \pm 1.5$ \\
Patients $(\mathrm{N}=4)$ & Dementia diagnosis & \\
& Alzheimer's & 50.0 \\
& Vascular & 25.0 \\
& Mixed & 25.0 \\
& SIRS score & $2.0 \pm 1.9$ \\
& Tube feeding (\%) & 75.0 \\
& Advance Care Planning completed $(\%)$ & 25.0 \\
\hline Caregivers $(\mathrm{N}=4)$ & Age (years) & $52.0 \pm 10.6$
\end{tabular}




\begin{tabular}{|c|c|c|}
\hline & Gender (female) & 50.0 \\
\hline & Educational level (\%) & \\
\hline & $<6$ years & 0 \\
\hline & $>6$ years & 100.0 \\
\hline & Relationship (\%) & \\
\hline & Spouse & 0 \\
\hline & Adult child & 100.0 \\
\hline & Co-Residency (\%) & 25.0 \\
\hline & Participate in the Namaste Care program (\%) & 50.0 \\
\hline \multirow{11}{*}{$\begin{array}{l}\text { Namaste Carers } \\
(\mathrm{N}=6)\end{array}$} & Age (years) & $32.3 \pm 5.6$ \\
\hline & Female (\%) & 100.0 \\
\hline & Nationality (\%) & \\
\hline & Singaporean & 16.7 \\
\hline & Filipino & 83.3 \\
\hline & Job description (\%) & \\
\hline & Senior staff nurse & 50.0 \\
\hline & Staff nurse & 33.3 \\
\hline & Enrolled nurse & 16.7 \\
\hline & Years employed in the healthcare & $8.7 \pm 3.8$ \\
\hline & Years of experience in dementia care & $4.1 \pm 1.5$ \\
\hline
\end{tabular}

SIRS = Severe Impairment Rating Scale

Note: Mean + SD unless otherwise indicated

A trend toward better cognitive response in PWAD post-program compared to pre-program with improving scores on SIRS (2.0 \pm SD 1.9 vs. $4.0 \pm$ SD 2.5) was evident. Comparison of QUALID scores pre- and post-program indicated a trend toward a better quality of life for PWAD after attending Namaste Care, as was evident by the decline in scores (29.0 \pm SD 7.4 vs. $24.0 \pm$ SD 4.3). The comparison of items in QUALID pre- and post-program also revealed the perception of PWAD enjoying being touched and interacting with others, being calmer, and more comfortable after Namaste Care (see Table 2). These findings were extrapolated further through the analysis of interview transcripts under qualitative results.

The mean score on qPAD Knowledge Test for the six Namaste Carers before the start of the Namaste Care program was $17.2(S D=2.0)$ with a maximum of 23 , where all carers scored well on questions addressing the prevention of negative outcomes in PwAD. The Namaste Carers maintained a high mean score (17.0 \pm SD 2.9) on the Knowledge Test on GPAD at least one-month after administering the Namaste Care program. They consistently answered correctly the questions such as;

"When a person is resistant to 'hands-on' care, it is best to stop what you are doing and come back later to try to complete the task" and "Physical restraints decrease the chance that a person with advanced dementia will fall" (Table 2). The Namaste Carers also reported high mean scores on the qPAD Attitude Scale pre- Namaste Care program (45.3 \pm SD 5.2) and continued showing such high scores post-program (44.3 \pm SD 3.4), indicating positive attitudes toward palliative care for advanced dementia. 
Table 2 Comparison of QUALID and qPAD items in pre- and post- Namaste Care.

\begin{tabular}{|c|c|c|c|}
\hline & Items & $\begin{array}{l}\text { Pre-Namaste Care } \\
\text { (mean score) }\end{array}$ & $\begin{array}{l}\text { Post-Namaste Care } \\
\text { (mean score) }\end{array}$ \\
\hline \multirow{4}{*}{ QUALID } & $\begin{array}{l}\text { PWAD has a facial expression of } \\
\text { discomfort-appears unhappy or in pain }\end{array}$ & 2.75 & 2.00 \\
\hline & PwAD enjoys touching/being touched & 3.25 & 2.00 \\
\hline & $\begin{array}{l}\text { PwAD enjoys interacting or being with } \\
\text { others }\end{array}$ & 3.25 & 2.25 \\
\hline & $\begin{array}{l}\text { PwAD appears emotionally calm and } \\
\text { comfortable }\end{array}$ & 2.25 & 1.75 \\
\hline \multirow{4}{*}{$\begin{array}{l}\text { qPAD } \\
\text { Attitudes }\end{array}$} & $\begin{array}{l}\text { On most days, I am satisfied with my job } \\
\text { of caring for PwAD }\end{array}$ & 3.66 & 3.83 \\
\hline & $\begin{array}{l}\text { My input and opinion are valued } \\
\text { regarding the needs of PWAD }\end{array}$ & 3.66 & 3.83 \\
\hline & $\begin{array}{l}\text { On most days, I feel I am part of the } \\
\text { care team }\end{array}$ & 4 & 4 \\
\hline & & (\% correct) & (\% correct) \\
\hline \multirow{3}{*}{$\begin{array}{l}\text { qPAD } \\
\text { Knowledge }\end{array}$} & $\begin{array}{l}\text { When a person is resistant to "hands- } \\
\text { on" care, it is best to stop what you are } \\
\text { doing and come back later to try to } \\
\text { complete the task. }\end{array}$ & 83.3 & 100 \\
\hline & $\begin{array}{l}\text { Physical restraints decrease the chance } \\
\text { that a PWAD will fall }\end{array}$ & 83.3 & 100 \\
\hline & $\begin{array}{l}\text { PWAD cannot really convey or relate to } \\
\text { caregivers if they are hungry, have pain, } \\
\text { or need to use the bathroom }\end{array}$ & 100 & 100 \\
\hline
\end{tabular}

Note: PwAD = person with advanced dementia;

QUALID = Quality of Life in Late-Stage Dementia;

qPAD = Questionnaire on Palliative Care for Advanced Dementia;

Higher qPAD Attitudes scores denote positive attitude toward PWAD; Lower QUALID scores denote a higher quality of life 


\subsection{Qualitative Results}

Three main themes emerged from the thematic analysis of ten interview transcripts from the caregivers and Namaste Carers: (1) the polarizing paradigm of care in advanced dementia; (2) preeminence of Asian family values; and (3) small actions make a big difference (Table 3).

Table 3 Major Themes identified by thematic analysis of 10 interview transcripts from Caregivers and Namaste Carers.

\begin{tabular}{ll}
\hline Themes & Sub-themes \\
\hline $\begin{array}{l}\text { Polarizing paradigm of } \\
\text { care in advanced dementia }\end{array}$ & $\begin{array}{l}\text { Biomedical-centric versus person-centric care approaches; } \\
\text { hospital-based versus home-based interventions at the end-of- } \\
\text { life }\end{array}$ \\
\hline Pre-eminence of Asian & $\begin{array}{l}\text { Involvement of family as a cohesive unit; } \\
\text { family values }\end{array}$ \\
& $\begin{array}{l}\text { Family togetherness and strength of relationships; } \\
\text { Catalyst for active participation in care provision; } \\
\text { Positivity in perspective }\end{array}$ \\
\hline Small actions make a big & $\begin{array}{l}\text { Small act of acknowledging personhood to deliver person- } \\
\text { difference }\end{array}$ \\
& $\begin{array}{l}\text { centered care; } \\
\text { Recognition that small gestures can reap huge benefits; }\end{array}$ \\
& Identifying and responding to micro to effect macro changes \\
\hline
\end{tabular}

\subsubsection{Polarizing Paradigm of Care in Advanced Dementia}

Caregivers expressed polarizing views of treatment for PWAD. The management at the end-oflife for PWAD entailed making the patient feel comfortable through avoidance of unnecessary interventions, yet many PWAD were not comfortable with the lack of biomedical intervention. The families struggled with the notion of seeking treatment for medical issues yet understood the need for PWAD to be in the company of their loved ones at home, within a familiar care environment.

One caregiver-09 expressed his thoughts as follows:

"The whole idea about not treating and just making patients feel more comfortable toward the later stages of life, I think, is not easily acceptable by the Asians, in the Asian community, so maybe...We can do something about that idea and make the caregivers understand the actual concept of keeping a patient comfortable and happy";

"That is the Asian mindset, you know, if you are sick, you should be in a hospital. Okay, you should be taken care of. But I think compared to; I don't know, Western thoughts; you think that is fine; a patient is approaching their certain state; then he should be better; be with the same person he loves most rather than be in a hospital or an intensive care unit."

The caregivers also struggled with the interpretations of QOL for their loved ones with advanced dementia. They endeavored to make comfort the main goal of end-of-life care. At the same time, they perceived their loved ones to have a poor QOL once they were in the advanced stage despite their attempts at delivering compassionate care.

Caregiver-11 aired her emotions as under: 
"We, family members try to make them as comfortable as we can and comfortable in our understanding meaning-making (to be) sure ... you know in terms of heat, temperature, in terms of food and liquid intake, in terms of the furniture ... I think the physical aspect, the physical comfort, we try our very best. But I can't speak for my mother because she is not able to move on her own. Certainly, that is not quality of life while we try to exercise her, basic moving of the limbs or some stretching and bending, some massaging. I think if I am my mother, in term(s) of quality of life, you know if you are fully demented (sic) there is no quality of life to talk of."

Namaste Carers adopted a more person-centric care approach toward dementia care through a better understanding of PWAD and establishing a good rapport with family caregivers. This is evident in the approach highlighted here below by Namaste Carer-03:

"Because you're really into the program, you really have to get to know your patient. For the family, you really have to make a rapport with them. So, talk to them closely compared to other patients, and I mean, you really have to get to know them. Talk to them and ask them what their feelings and thoughts are. You really have this close relationship."

To these healthcare professionals, the PWAD felt fulfillment when care was individualized, and patients' preferences were respected.

Namaste Carer-07 opined as under:

"We need to be patient and to know what is the patient's preference, as well as of (family) carers ... the task is fulfilling."

\subsubsection{Pre-eminence of Asian Family Views}

The caregivers viewed the family as a cohesive unit that served as a vehicle in the delivery of the Namaste Care program. Members of the family were encouraged to participate in the program with unity in the numbers toward delivering holistic care for PWAD.

"More participants would be better. And the more, the merrier. So, if the family can come in and join, it is even better." (Caregiver-05)

Family participation alongside care staff in Namaste Care became a reason why family members gathered and anticipated the sessions enthusiastically. The program provided an opportunity to spend quality time with their loved ones. One of the Namaste Carers noticed that the PWAD involved in the Namaste Care program had received good family support. Closer relationships, mediated through loving touch, were forged and strengthened between caregivers and PWAD through Namaste Care sessions. Mutual bonds were also cemented through non-verbal communications, in which care was delivered in a loving and patient manner.

"It gives more time for bonding because everybody sits together and does it together." (Caregiver-09)

Namaste Care served as a catalyst for the involvement of other family members in the provision of care toward PWAD, where the program constructed a structure for them to build upon and emulate at home.

"I would say actually, without her coming into this situation now, none of us would have been paying more attention. Sometimes it takes something like this to get people to help. It has actually brought forth a momentum for us to work." (Caregiver-05)

Caregiver-07 quipped on the momentum to work: "Very encouraging for us, the family members." 
Namaste Carers also observed the ease with which caregivers could learn the myriad of sensory interventions performed during Namaste Care with the hope of continuing such care at home.

Positivity in perspective or outlook permeated through the family units as caregivers and other family members experienced the benefits of the program.

"Any improvement in my mother will have a positive impact on me. And also, on the relationship, between mother and us. And also, on the family, our families..." (Caregiver-07)

Caregivers felt comfortable with the existence of the Namaste Care program. Their loved ones with advanced dementia could still receive personalized and tailored care, which enabled them to view dementia care at the end-of-life positively.

"Even though...there are no significant changes, I did experience the comfort that my mother is undergoing such a program." (Caregiver-11)

\subsubsection{Small Actions Make a Big Difference}

Person-centered care in Namaste Care entails the provision of persevering, individualized, sensorial, and micro care. Through the small act of understanding and knowing the person behind the disease, a healthy caregiver-healthcare staff alliance can be forged. Namaste Carers succeeded in building a good rapport and established an implicit understanding of care for PWAD.

"It helps me know patients better and then know what they like or dislike, because we also give them food and so we know.... we will get to know the patients better, in-depth and we have more time spending (sic) with the patients and also the carer... knowing the carer." (Namaste Carer-04)

Another Caregiver stated as under:

"In terms of relationship with the caregiver and the relative of the patient, we really establish a great rapport. Some of them really are looking forward to that; what is the Namaste care session, what time is it, can we go in, can we interact with the patient when we are doing the Namaste care. It really makes a good relationship with them like the trust is really built, and they really can rely on you as well." (Namaste Carer-09)

Namaste Carers also acknowledged and recognized that their small acts or gestures of kindness under Namaste Care were able to reap huge benefits. The moments of recognition can be epiphanic and fulfilling. These affective experiences might be important for persevering in what was conceivably a very tough care provision in healthcare.

"I can just contribute ...even just for a simple task for them like doing some...therapy at least the patient can appreciate... when we (are) doing some massage. I can contribute to the family members even in such a small period of time." (Namaste Carer-01)

A Namaste carer had a sense of fulfillment when PwAD responded well during the Namaste Care sessions and enjoyed the therapeutic interventions.

"You feel complete also when you take care like... when you groom them and when you massage (them)." (Namaste Carer-05)

The emphasis on the small means that professional and family caregivers have to be trained to notice and respond to the micro care, in order to achieve macro effects, such as good quality care and quality of life for PWAD.

"For some of our patients here, I think after one or two sessions of massaging and stimulating their five senses, they can even barely move their upper arms, they can even move their lower 
limbs even the toes ... that little improvement you see in your patient really gives you a sense that you are doing something good." (Namaste Carer-09)

\subsection{Namaste Care as A Bridge}

Namaste care serves as a bridge to fill the gap between the biomedical model of dementia care and person-centered care on the premise that small actions make a big difference and lead to an improved QOL and cognitive responses in PWAD (Figure 1). The program can be delivered by healthcare staff having good knowledge and a positive attitude toward dementia care and the family members, as a cohesive unit or in collaboration with healthcare staff. It provides family caregivers a tangible way of meaningful participation (at a personal level and with family involvement) that corresponds with the Asian family values, brings comfort, and alleviates the sense of loss and alienation even though the biomedical aspects remain largely unchanged. When equipped with adequate knowledge and positive attitudes toward advanced dementia preprogram, healthcare staff could deliver person-centered care to PWAD, enriched their knowledge, and displayed positive attitudes toward PWAD post-program.

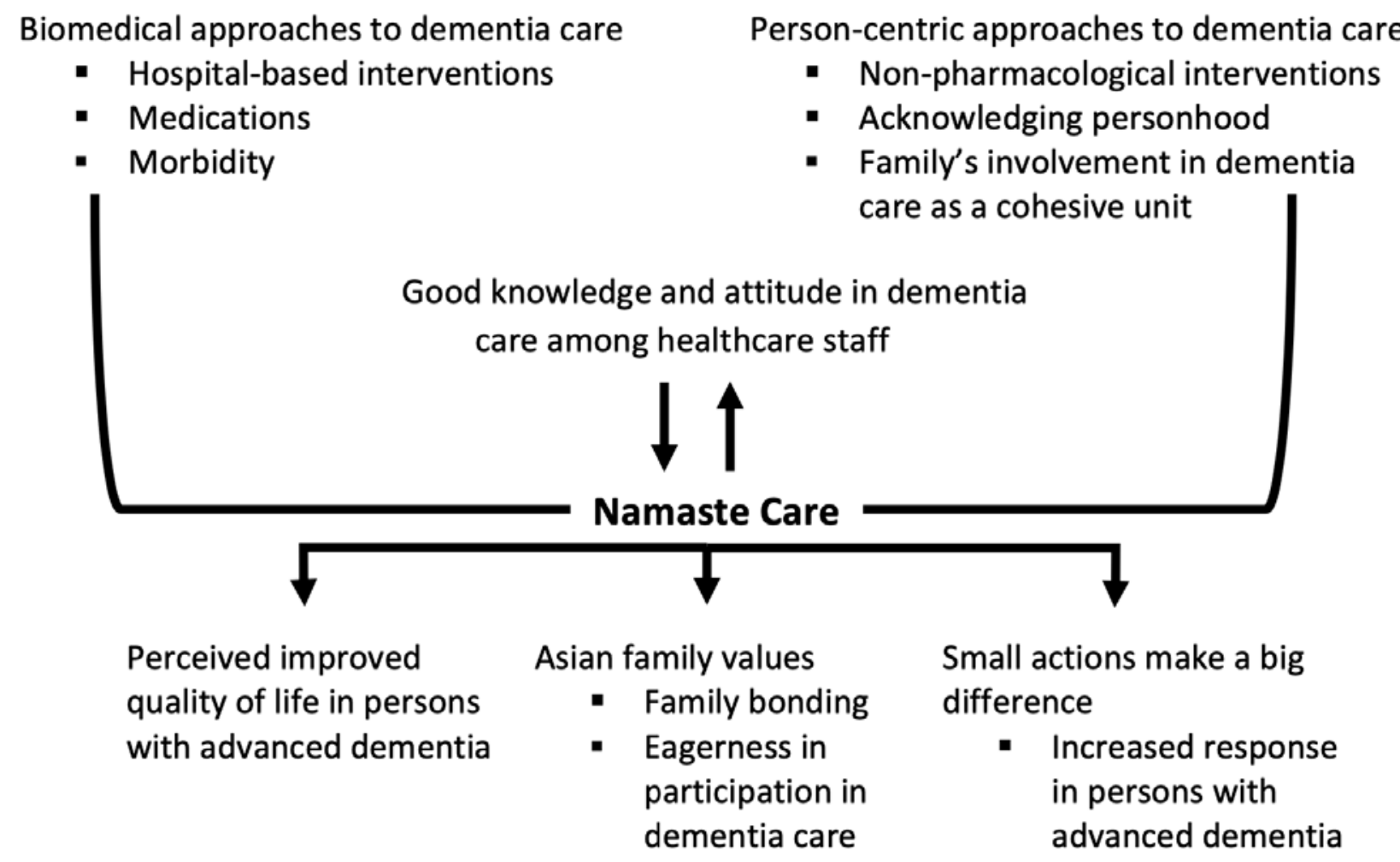

Figure 1 Influence of Namaste Care on patient-caregiver dyads and Namaste Carers.

\section{Discussion}

The current study highlighted the need for polarizing paradigm of care in advanced dementia and showed how Namaste Care may serve as a bridge between the opposing biomedical-centric and person-centric care approaches. This study also considered the importance of the Asian family values in molding the perspective and views of caregivers for PWAD on providing care in the advanced stage of dementia through Namaste Care while the major theme of small actions make 
a big difference emphasized the notion that small effort in delivering person-centered care can lead to huge benefits. Qualitative findings from our study supported the quantitative results of a positive trend toward the better-perceived QOL for PWAD and improved cognitive responses with Namaste Care.

The family caregivers expressed conflicting views on the optimization of care for PWAD, with preponderance toward adopting a biomedical-centric approach. These polarizing views could be explicated by the Asian values of family cohesion and deference to healthcare professionals [39]. The Asian values of family cohesion frequently place the needs of the group over the individual; thus, the evident tension in decision-making between seeking treatment in the hospital or being at home as the family experienced unprepared in accepting end-of-life care for PWAD. The implicit trust in healthcare professionals with respect for persons of authority drove caregivers into utilizing hospital services for their loved ones where they perceived optimized care. This healthcare-seeking behavior and administration of invasive interventions in the hospital may pose a challenge with the goals of care in the advanced stage of dementia (when more focus on comfort, dignity, and better quality of life is desirable) [40]. In contrast, Namaste Carers, who were healthcare professionals, displayed a tendency toward adopting person-centric care approaches, which was evident by their endorsement of the theme: small actions make a big difference. There is the quality of life for PWAD if we can look away from our tinted biomedical lenses to see that their needs for humanistic person-centered care remains as germane and pressing as ever. This paradoxical observation illuminates the tension between the biomedical-centric and personcentric model of dementia care. It also highlights the critical role played by Namaste Care in bridging the cleft between these two opposing approaches and evolving a converged combined approach for better outcomes. The polarizing paradigm of care represents the meaning-making of family caregivers and Namaste Carers about the philosophy of care approaches in advanced dementia.

The current study endorsed the pivotal role that the family unit plays in healthcare delivery for persons with dementia, which may shape the perception of caregivers about Namaste Care. Asian family values place family relationships at the core of the conduct of healthcare practices. Family values and virtues are the principal points at which Asian societies construct the concept of self [41], where "socio-centric construction of personhood and decision-making" prevails [42]. The notion of collective good over an individual is pervasive in Asian cultures, and cultural factors play a vital role in influencing the perceptions of caregiving [43]. The emotional and deep involvement of the family in Namaste Care in shared decision making on treatment and end-of-life care [44] contrasts that of Western society's emphasis on individual autonomy in the biomedical approach. Care provisions in Asian healthcare settings tend to adopt a family-centric model [45] where family participation is sought and is an inherent part of care norms [46]. Family members are perceived as partners in dementia care [30], and the locally adapted Namaste Care program facilitates the family's involvement in the provision of such care.

Caregivers in the current study underscored the benefits that could accrue to the family members through participation in Namaste Care, such as being more involved in the care of their loved ones with advanced dementia and bonding through the delivery of meaningful activities to PWAD. These findings are in line with previous ones $[47,48]$ where Namaste Care resulted in the strengthening of family relationships by the empowerment of families to interact and engage with PwAD. Moreover, Walmsley and McCormack [49] documented how family caregivers for PWAD 
sought solace in family groups for a meaningful engagement "rooted in familial bonds, trust, and familiarity". Stacpoole et al. (2017) demonstrated how families reconnected with their loved ones suffering from dementia and reclaimed a meaningful role in their lives through Namaste Care [9]. A study conducted in a Canadian Setting [50] explored how the families become more engaged in caring when their loved ones are enrolled in Namaste Care, and they feel empowered, encouraged participating in dementia care. Although these studies were conducted in Western societies by the non-Asian caregivers, the commonality in experience with Namaste Care between both the Asian and the Western societies was the integration of person-centered care approaches into the conduct of various intervention programs. Asian culture not only emphasizes the significant role of a family unit but also gives weightage to familial kinships and strong family ties [39]. Namaste Care may be well received in Asian societies as it is built on healthy relationships, fosters sensitivity and mutual respect, and addresses the inherent consistencies between Asian values and the core values of person-centered care underpinning the novel Namaste Care.

Healthcare providers experience tension between completing nursing tasks or documentation and the desire to attend to the needs of PwAD. There are less fruitful interactions as care becomes focused on the completion of tasks in a methodical rather than purposeful manner. The demands of a higher level acuity care amid constraints of time stand against the provision of personalized care in most care settings. Notwithstanding, the theme of small actions make a big difference in Namaste Care can perpetuate the small acts of acknowledging personhood and minute gestures of delivering individualized care into defining a new standard of quality care for PwAD.

In this study, the Namaste Carers acknowledged the role of their perceived small acts in producing huge benefits through the program. Their attempts at understanding the person behind the disease allowed for personalization of therapeutic interventions during Namaste Care sessions and enabled PWAD to feel a sense of comfort. Their knowledge of advanced dementia and positive attitudes toward PWAD was reflected in the high scores on GPAD, which corroborate the findings from a local study [51] that revealed a tendency of people/carer with good knowledge and attitude toward dementia to understand the implications of the dreaded disease. Simple individualized physical touches during Namaste Care sessions were intentional and purposeful, compared to the functional touches delivered through conventional care. The care was focused on "being" rather than "doing", thereby mitigating the strong predisposition toward a task-oriented approach in conventional care. Several studies have highlighted the need of more responsiveness among the care recipients of Namaste Care with improved interactions such as positive vocalizations, making eye contact, and smiling $[20,50,52]$. QOL is thus not just about meeting physical needs but also about being lovingly touched and not just during care [52].

Namaste Care can be perceived as providing opportunities for Asian families to "develop literacy for communicating love and care" [53] as such expressions tend to be conservative and conducted through small acts of kindness. Understanding of small gestures, verbal and non-verbal cues of PWAD, in their attempts at communication due to underlying cognitive and functional deficits, involves going beyond the literal meaning with attempts at interpreting these gestures. These types of efforts represent one of the six access routes to gain insight into the subjective world of persons with dementia, as reported by Kitwood, a pioneer of person-centered care [10]. Ames [54] presented a similar lens to reflect the theme 'small actions make a big difference': the simple recognition of personhood, dignity, and unconditional worth required to epitomize the 
practice of person-centered care. Human disconnect and touch widen as dementia progresses globally with age despite the growing need for connection [55] and volition declining with the advancement of dementia. Namaste Care provides a suitable shared platform to engender a sense of connectedness among PWAD and care providers. In rendering compassionate care for PWAD, the fulfillment of one of the needs (such as attachment, inclusion, comfort, identity, occupation) of PWAD as described by Kitwood [10] results in the fulfillment of other needs to some extent. A small effort in ensuring and providing comfort and maintaining personhood may enable PwAD to achieve a good QOL. This finding in our study corroborates a Canadian study finding that indicated the need for improvement in the QOL through a reduction in QUALID scores [48].

The sub-themes of "small actions make a big difference" resonate well with the senses framework [56] of providing good quality care whereby both caregivers and Namaste Carers could experience caring relationships that promote a sense of purpose, significance, and achievement. The multitude of deficits experienced by the PwAD limits their involvement in decision-making and care plans. Namaste Care offers a voice to the patient and a potential vehicle to enable carers' active participation in care through minute responses with resultant significant effects. Therefore, Namaste Care can be implemented in real-world conditions within the hospital setting where healthcare providers need to juggle the constraints of time and administer care in proportion to the level of acuity. Proper staff training and innovative advanced dementia programs such as Namaste Care are mutually cohesive. Hence, the success of implementation would depend on PWADs' participation and satisfaction, family understanding, and healthcare providers' skill sets [57].

The strength of this study lies in the involvement of family caregivers in participating in Namaste Care sessions in collaboration with healthcare staff, thereby cementing a holistic therapeutic alliance. This local adaptation of the Namaste Care program provides a novel approach to how Namaste Care can be implemented in Asian communities. Smaling et al. alluded to Namaste Care and designed a study to evaluate the effects of the Namaste Family Care program on PWAD in long-term care homes [21]. Also, family caregivers verbalized enthusiasm in emulating the Namaste Care sessions at home; thus, advocating for home-based nonpharmacological interventions for PwAD. Matching Namaste Care to the supportive home environment will foster innovative caregiver education programs and reinforcement of personcentric care approaches in dementia care. Importantly, the successful delivery of the Namaste Care program for PWAD in the step-down unit was attributed to the presence of motivated and driven healthcare staff, as evidenced by the high attitude scores on qPAD who had volunteered as Namaste Carers. To ensure the sustainability of this program, non-medical volunteers were also recruited and adequately trained to deliver Namaste Care in the step-down unit. Future implementation of a similar locally-adapted Namaste Care program within the hospital setting may warrant a pool of medical and non-medical volunteers as Namaste Carers, in close collaboration with family caregivers.

Undoubtedly, the intersection of good culture and dementia care highlights the importance of cultural influences in understanding dementia and how care is provided for PWAD [58]. Additional studies are suggested to evaluate the impact of Namaste Care on Asian families and Asian healthcare providers, considering social and cultural norms that are unique in different Asian communities. Although the core principles of person-centered care are not culturally specific and are globally relevant, a need is still felt to consider the social and cultural contexts in the 
implementation of person-centered care through Namaste Care in our daily clinical practice [46]. Finally, research into the relationship between caregiver burden in dementia care and implementation of Namaste Care merits further consideration to better inform care providers of the role of Namaste Care in tackling challenging aspects of dementia care giving.

There were several notable limitations in this study. The sample size was too small for meaningful inferential statistical analysis; hence, we reported only a descriptive analysis of the emerging trend toward an improved cognitive response and quality of life scores. The study design was focused on data collection and assessment at two-time points (pre- and post-Namaste Care), which precludes meaningful conclusions about the sustainability of benefits of the post-Namaste Care program. The enrolment of Namaste Carers of diverse background may add to the richness of the qualitative data collected since the Namaste Carers in the present study comprised solely of healthcare providers from nursing service, which may provide a more uniform and homogeneous perspective of dementia care.

\section{Conclusions}

Overall, Namaste Care can be viewed as the microcosm of good quality care for persons with advanced dementia. It outlines a framework for the planning of meaningful activities in order to establish a new structured routine, away from conventional care, which tends to be task-oriented. Person-centered care strategies and interventions can be delivered through Namaste Care, which may serve to facilitate individualized care according to preferences and responses of persons with dementia. The development of Namaste Care programs among Asian societies needs to consider family's participation in the dementia care continuum, and its implementation approach needs to be culturally specific.

\section{Acknowledgments}

We wish to express our sincere gratitude to the nurses in the step-down unit for the support of Namaste Care, Nurse Clinician Lim Meow Gek for her valuable input in the conduct of the program and all participants of this study who have shared their experiences readily. Many thanks to Delwyn Lim for transcribing the audiotaped interviews verbatim.

\section{Author Contributions}

NA conceptualised, designed and planned the study. CC and CNT assisted in collecting the data. APC and JK interviewed the subjects. NA, ML and WSL analysed the data. All authors contributed to writing of the manuscript.

\section{Funding}

Nil funding.

\section{Competing Interests}

The authors have declared that no competing interests exist. 


\section{References}

1. Kitwood T. Brain, mind and dementia: With particular reference to Alzheimer's disease. Aging Soc. 1989; 9: 1-15.

2. Bond J. The medicalization of dementia. J Aging Stud. 1992; 6: 397-403.

3. Lawton MP, Rubinstein RL. Interventions in dementia care: Toward improving quality of life. New York: Springer Publishing Company; 2000.

4. Downs M, Clare L, Anderson E. Dementia as a biopsychosocial condition: Implications for practice and research. Handbook of the clinical psychology of ageing. 2nd edHoboken, New Jersey: John Wiley \& Sons; 2008. 145p.-159p.

5. Hsieh PC, Wu SC, Fuh JL, Wang YW, Lin LC. The prognostic predictors of six-month mortality for residents with advanced dementia in long-term care facilities in Taiwan: A prospective cohort study. Int J Nurs Stud. 2019; 96: 9-17.

6. Hum AY, Wu HY, Ali NB, Leong IY, Chin JJ, Lee AO, et al. The dignity in advanced dementia (diadem) study: Developing an integrated geriatric palliative homecare program. Prog Palliat Care. 2018; 26: 65-72.

7. Morgan S, Yoder LH. A concept analysis of person-centered care. J Holist Nurs. 2012; 30: 6-15.

8. Simard J. The end-of-life Namaste Care program for people with dementia. 2nd ed. Baltimore, Maryland: Health Professions Press, Inc; 2013.

9. Stacpoole M, Hockley J, Thompsell A, Simard J, Volicer L. Implementing the Namaste Care program for residents with advanced dementia: Exploring the perceptions of families and staff in UK care homes. Ann Palliat Med. 2017; 6: 327-39.

10. Kitwood T. The experience of dementia. Aging Ment Health. 1997; 1: 13-22.

11. Fullarton J, Volicer L. Reductions of antipsychotic and hypnotic medications in Namaste Care. J Am Med Dir Assoc. 2013; 14: 708-709.

12. Duffin C. How Namaste principles improve residents' lives: Christian Duffin describes how older people in a home in Kent are benefiting from an American programme that uses sensory techniques to comfort and soothe them. Nurs Older People. 2012; 24: 14-18.

13. Stacpoole M, Hockley J, Thompsell A, Simard J, Volicer L. The Namaste Care programme can reduce behavioural symptoms in care home residents with advanced dementia. Int J Geriatr Psychiat. 2015; 30: 702-709.

14. Simard J, Volicer L. Effects of Namaste Care on residents who do not benefit from usual activities. Am J Alzheimers Dis Other Demen. 2010; 25: 46-50.

15. Thompsell A, Stacpoole M, Hockley J. Namaste Care: The benefits and challenges. J Dement Care. 2014; 2: 28-30.

16. Volicer L. Namaste Care improves quality of life for dementia patients. Today Geriatr Med. 2016; 9: 5.

17. Bray J, Brooker DJ, Garabedian C. What is the evidence for the activities of Namaste Care? A rapid assessment review. Dementia. 2019; 1471301219878299.

18. Manzar B, Volicer L. Effects of Namaste Care: Pilot study. Am J Alzheimers Dis. 2015; 2: 24-37.

19. Mead N, Bower P. Patient-centredness: A conceptual framework and review of the empirical literature. Soc Sci Med. 2000; 51: 1087-1110.

20. McNiel P, Westphal J. Namaste Care ${ }^{\mathrm{TM}}:$ A person-centered care approach for Alzheimer's and advanced dementia. West J Nurs Res. 2018; 40: 37-51. 
21. Smaling JH, Joling KJ, Van de Ven PM, Bosmans JE, Simard J, Volicer L, et al. Effects of the Namaste Care Family programme on quality of life of nursing home residents with advanced dementia and on family caregiving experiences: Study protocol of a cluster-randomised controlled trial. BMJ open. 2018; 8: e025411.

22. Bunn F, Lynch J, Goodman C, Sharpe R, Walshe C, Preston N, et al. Improving living and dying for people with advanced dementia living in care homes: A realist review of Namaste Care and other multisensory interventions. BMC geriatr. 2018; 18: 303.

23. Nicholls D, Chang E, Johnson A, Edenborough M. Touch, the essence of caring for people with end-stage dementia: A mental health perspective in Namaste Care. Aging Ment Health. 2013; 17: 571-578.

24. Froggatt K, Patel S, Algorta GP, Bunn F, Burnside G, Coast J, et al. Namaste Care in nursing care homes for people with advanced dementia: Protocol for a feasibility randomised controlled trial. BMJ open. 2018; 8: e026531.

25. Froggatt K, Walshe C, Burnside G, Perez Algorta G, Kinley J, Preston N HB et al. The Namaste Care intervention to improve the quality of dying for people with advanced dementia living in care homes: A realist review and feasibility study for a cluster randomised controlled trial. BMJ Open. 2018; 8: e026531.

26. Froggatt K, Best A, Bunn F, Burnside G, Coast J, Dunleavy L, et al. A group intervention to improve quality of life for people with advanced dementia living in care homes: The Namaste feasibility cluster RCT. Health Technol Assess 2020; 24: 1-140.

27. John K, Koffman J. Introducing Namaste Care to the hospital environment: A pilot study. Ann Palliat Med. 2017; 6: 354-364.

28. Chen YC. Chinese values, health and nursing. J Adv Nurs. 2001; 36: 270-273.

29. Dewi WN, Evans D, Bradley H, Ullrich S. Person-centred care in the Indonesian health-care system. Int J Nurs Pract. 2014; 20: 616-622.

30. Chan SW. Family caregiving in dementia: The Asian perspective of a global problem. Dement Geriatr Cogn Disord. 2010; 30: 469-478.

31. Reisberg B, Ferris SH, Franssen E. An ordinal functional assessment tool for Alzheimer's-type dementia. Hosp Community Psychiatry. 1985; 36: 593-595.

32. Charlson ME, Pompei P, Ales KL, MacKenzie CR. A new method of classifying prognostic comorbidity in longitudinal studies: Development and validation. J Chronic Dis. 1987; 40: 373383.

33. Rabins PV, Steele CD. A scale to measure impairment in severe dementia and similar conditions. Am J Geriatr Psychiatry. 1996; 4: 247-251.

34. Yeo C, Lim WS, Chan M, Ho XQ, Anthony PV, Han HC, et al. Severe impairment rating scale: A useful and brief cognitive assessment tool for advanced dementia for nursing home residents. Am J Alzheimers Dis Other Demen. 2016; 31: 87-96.

35. Weiner MF, Martin-Cook K, Svetlik DA, Saine K, Foster B, Fontaine CS. The quality of life in late-stage dementia (QUALID) scale. J Am Med Dir Assoc. 2000; 1: 114-116.

36. Chen IH, Lin KY, Hu SH, Chuang $\mathrm{YH}$, Long $\mathrm{CO}$, Chang $\mathrm{CC}$, et al. Palliative care for advanced dementia: Knowledge and attitudes of long-term care staff. J Clin Nurs. 2018; 27: 848-858.

37. Long CO, Sowell EJ, Hess RK, Alonzo TR. Development of the questionnaire on palliative care for advanced dementia (qPAD). Am J Alzheimers Dis Other Demen. 2012; 27: 537-543.

38. Braun V, Clarke V. Using thematic analysis in psychology. Qual Res Psychol. 2006; 3: 77-101. 
39. Bui YN, Turnbull A. East meets west: Analysis of person-centered planning in the context of Asian American values. Educ Trai Dev Disabil. 2003; 38: 18-31.

40. Volicer L. Goals of care in advanced dementia: Quality of life, dignity and comfort. J Nutr Health Aging. 2007; 11: 481.

41. Kong ST, Fang CM, Lou VW. Organizational capacities for 'residential care homes for the elderly' to provide culturally appropriate end-of-life care for Chinese elders and their families. J Aging Stud. 2017; 40: 1-7.

42. Seeberg J, Verma K, Mehta M, Dutta R, Addlakha R. Family autonomy and patient rights to healthcare in an 'Asian values' context. Folk. 2004; 45: 87-104.

43. Wang DS. Caregiving for dementia in Asian communities: Implications for practice. J Ethn Cult Divers Soc Work. 2012; 21: 249-473.

44. Phua J, Kee AC, Tan A, Mukhopadhyay A, See KC, Aung NW, et al. End-of-life care in the general wards of a Singaporean hospital: An Asian perspective. J Palliat Med. 2011; 14: 12961301.

45. Kwak J, Haley WE. Current research findings on end-of-life decision making among racially or ethnically diverse groups. Gerontologist. 2005; 45; 634-641.

46. Wang J, Wu B, Bowers BJ, Lepore MJ, Ding D, McConnell ES, et al. Person-centered dementia care in China: A bilingual literature review. Gerontol Geriatr Med. 2019; 5: 2333721419844349.

47. Thompsell A, Stacpoole M, Hockley J. Namaste Care: The benefits and challenges. J Dement Care. 2014; 2: 28-30.

48. Kaasalainen S, Hunter PV, Dal Bello-Haas V, Dolovich L, Froggatt K, Hadjistavropoulos T, et al. Evaluating the feasibility and acceptability of the Namaste Care program in long-term care settings in Canada. Pilot Feasibility Stud. 2020; 6: 1-2.

49. Walmsley B, McCormack L. Dementia: Aloneness, social and relational engagement, and psychological growth in families. OBM Geriatr. 2018; 2: 013.

50. Kaasalainen S, Hunter PV, Hill C, Moss R, Kim J, van der Steen JT, et al. Launching 'Namaste Care' in Canada: Findings from training sessions and initial perceptions of an end-of-life programme for people with advanced dementia. J Res Nurs. 2019; 24: 403-417.

51. Tan WJ, Hong SI, Luo N, Lo TJ, Yap P. The lay public's understanding and perception of dementia in a developed Asian nation. Dement Geriatr Cogn Dis Extra. 2012; 2: 433-444.

52. Simard J. Silent and invisible; nursing home residents with advanced dementia. J Nutr Health Aging. 2007; 11: 484-488.

53. Leung PP, Chan CL. Palliative care in the Chinese context: An integrated framework for culturally respectful practice. Oxford Textbook of Palliative Social Work. New York: Oxford University Press; 2011. p.573- p.578

54. Ames $\mathrm{S}$. The value given and presupposed in person-centered dementia care. OBM Geriatr. 2019; 3: 068.

55. Nicholls D, Chang E, Johnson A, Edenborough M. Touch, the essence of caring for people with end-stage dementia: A mental health perspective in Namaste Care. Aging Ment Health. 2013; 17: 571-578.

56. Nolan MR, Davies S, Brown J, Keady J, Nolan J. Beyond 'person-centred' care: A new vision for gerontological nursing. J Clin Nurs. 2004; 13: 45-53. 
57. Arrey SK. Commentary: Launching 'Namaste Care' in Canada: Findings from training sessions and initial perceptions of an end-of-life programme for people with advanced dementia. J Res Nurs. 2019; 24: 418-419.

58. Brooke J, Cronin C, Stiell M, Ojo O. The intersection of culture in the provision of dementia care: A systematic review. J Clin Nurs. 2018; 27: 3241-3253.

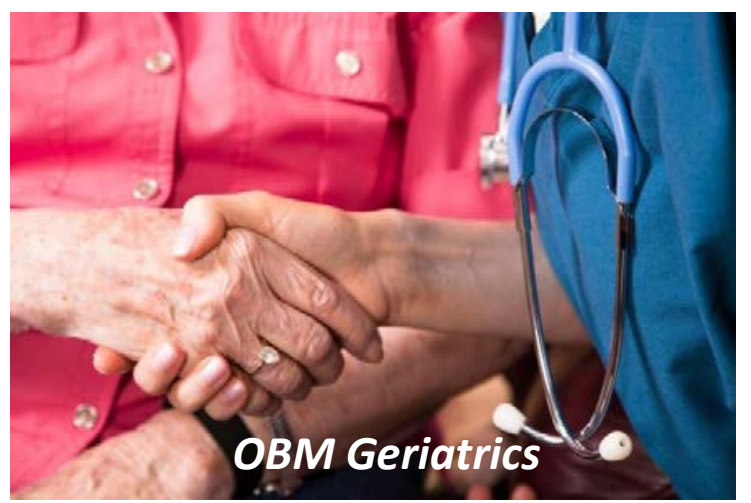

Enjoy OBM Geriatrics by:

1. Submitting a manuscript

2. Joining in volunteer reviewer bank

3. Joining Editorial Board

4. Guest editing a special issue

For more details, please visit: http://www.lidsen.com/journals/geriatrics 